\section{Histone mRNA and maternal message}

\section{from a Correspondent}

Histone mRNA not surprisingly may be isolated from many cell types as a 7-9S component which resolves on acrylamide gels into six or seven bands (Weinberg et al., Nature, 240, 225; 1972). Until recently the evidence in favour of these RNAs being truly histone messages was circumstantial, but Jacobs-Lorena, Baglioni and Borum (Proc. natn. Acad. Sci. U.S.A., 69, 2095; 1972) have provided proof that a $7-9 S$ RNA species is translated into histones in an ascites cell free system. The original work of Robbins and Borun (Proc. natn. Acad. Sci. U.S.A., 57, 409; 1967) showed that in HeLa cells histones are synthesised during $S$ phase on small cytoplasmic polyribosomes and this led to the identification of a rapidly labelled 9S RNA species associated with them (Borun, Scharff and Robins, Proc, natn. Acad. Sci. U.S.A., 58, 1977; 1967).

Early work by Kedes and Gross on sea urchin embryos (Nature, 223, 1335; 1969; and J. molec. Biol., 42, 559; 1969) confirmed the existence of a newly synthesised 9S RNA species associated with small polysomes during the phase of rapid cell division called cleavage at the start of development. The system was notable for the very high specific activity to which the 9S RNA could be labelled, for the apparent ease of $9 S$ RNA purification and for its freedom from contamination by labelled ribosomal RNA, which is not synthesised in appreciable quantities until much later. It is not difficult to think of things to do with highly labelled mRNAs even though they lack authentic credentials and, first, Kedes and Birnstiel (Nature new Biol., 230, 165; 1971), in what they regarded as the first steps to the isolation of a eukaryotic gene coding for a specific protein, produced evidence for reiterated, closely clustered histone coding sequences potentially separable from the rest of the genome.

Weinberg, Birnstiel, Purdom and Williamson (Nature, 240, 225; 1972) took this analysis further by showing that component species separable from 9S histone messages each hybridise to DNA sequences several hundred times repetitive. The different groups of repetitive histone DNA seem to be closely linked since hybridisation of the RNA subfractions to DNA banded in $\mathrm{CsCl}$ shows that they all fall in the same region at a density $\left(1.705 \mathrm{~g} \mathrm{cc}^{-1}\right)$ just greater than the main band $(1.697 \mathrm{~g}$ $\mathrm{cc}^{-1}$ ).

In spite of the fact that histone 9S mRNA synthesis starts soon after fertilisation in sea urchins, treatment with actinomycin $\mathrm{D}$ at concentrations which inhibit synthesis permits many cleavages and apparently normal development to the blastula stage (Gross and Cousineau, Exptl cell Res., 37, 368; 1964). Embryos thus treated continue to synthesise proteins though not nuclear proteins during this time (Raff, Greenhouse, Gross and Gross, J. cell Biol., $50,520 ; 1971)$ and this was regarded as indirect evidence for the absence of stored histone message in unfertilised eggs.

Two recent papers from Gross and his colleagues now provide direct proof that histone mRNA on the contrary is stored in unfertilised sea urchin eggs although at a lower level than that attained during cleavage, and this constitutes the first demonstration of a maternally-inherited message with a defined coding sequence. Many previous attempts have been made to provide direct evidence for stored maternal mRNA in echinoderm (Slater and Spiegelman, Proc. natn. Acad. Sci., U.S.A., 56, 164; 1966) and Xenopus (Davidson, Crippa, Kramer and Mirsky, ibid., 66, 379; 1972) embryos, but in none of these cases was an identifiable maternal RNA species shown to stimulate the synthesis of a defined product.

What Gross et al. (Proc. natn. Acad. Sci. U.S.A., 70, 2614; 1973) did was to extract RNA from different regions of a sucrose gradient in which a post mitochondrial supernatant from homogenised unfertilised echinoderm eggs had been centrifuged such that the $80 \mathrm{~S}$ ribosomes were almost pelleted. They found that a mouse ascites cell free system will make protein from these RNA preparations but that the most active fractions are from the ribosome subunit region of the gradient (2040S). Using aspartic acid and lysine to show up differences in mRNA coding sequence they found that the faster sedimenting RNP fractions give RNA which incorporates more aspartic acid relative to lysine than the more slowly sedimenting fractions. By running the labelled products in acrylamide gels together with authenic labelled histone, and by analysing tryptic peptides, they were able to show that the RNA from a slowly sedimenting (20S) RNP fraction makes histone protein almost exclusively and that the more rapidly an RNP sediments beyond $20 \mathrm{~S}$ the less histone protein will be made by its RNA. These experiments show that mRNAs exist in non-ribosome bound RNP particles from unfertilised sea urchin eggs and that they are capable of stimulating the synthesis of histone in a cell free protein synthesising system.

Skoultchi and Gross (Proc: natn. Acad. Sci., U.S.A., 70, 2840; 1973), using the completely different approach of competition-hybridisation to detect specific mRNA sequences, have been able to provide direct support for the conclusions drawn from in vitro protein synthesis studies. They used ${ }^{3} \mathrm{H}$-labelled 9S RNA from sea urchin embryos in conditions in which most of the input labelled RNA can form hybrids with DNA but which still allows identical unlabelled RNA to compete for sites. Both total RNA from small polysomes and unfertilised eggs competed in the reactions but purified 18 and $26 \mathrm{~S}$ ribosomal RNA, rabbit globin mRNA and 9S histone mRNA from another echinoderm species did not. The curves generated suggest that the amount of histone mRNA in unfertilised eggs is one-quarter of that in the small polysomes of an embryo of 100 cells. This figure is in agreement with similar work by Farquhar and McCarthy (Biochem. biophys. Res. Comm., 53, 515; 1973). who used very different conditions for their hybridisation.

Skoultchi and Gross went on to show that RNA from subribosomal RNP particles from unfertilised eggs competes with $9 \mathrm{~S}$ histone mRNA in agreement with the prediction from the protein synthesis studies of Gross et al. These papers represent a real advance since they unequivocally identify by two very different methods a messenger RNA which is maternally inherited.

\section{Role of plastocyanin in photosynthesis}

\section{from our Photosynthesis Correspondent}

THE old argument whether or not plastocyanin acts between cytochrome $f$ and the reaction centre of photosystem 1 (P700) now seems to be over. It has been known for many years that this copper containing protein is an important component of the photosynthetic electron transport chain but there have been several contradictory hypotheses about its exact site of action. The most widely accepted view is that plastocyanin acts as an intermediate between cyt $f$ and P700. Not all investigators, however, agree with this scheme and prefer to place plastocyanin on the oxidising side of cyt $f$ so that cyt $f$ acts as the primary electron donor to P700. Alternative suggestions have been that plastocyanin and cyt $f$ act in parallel in such a way that they both donate electrons to P700 or that they are associated with the different photosystems; plastocyanin with photosystem 2 and cyt $f$ with photosystem 1 .

It now seems from a recent publication from San Pietro's laboratory at Indiana (Siedow, Curtis and San Pietro, Arch. Biochem. Biophys., 158, 889; 1973) that the most popular hypothesis is correct. In a very careful piece of work Siedow et al. have demonstrated that plastocyanin acts stoichiometrically as an electron carrier between cyt $f$ and P700. Their work is particularly convincing because they have managed to 